there are no toxic substances, it is safe according to the criteria of ecological and hygienic human nutrition concept.

Antioxidant effects in the organism of white rats have been studied at the initiation of the processes of lipid peroxidation by the introduction of carbon tetrachloride. We were determined the products of lipid peroxidation for the evaluation of antioxidant effect in accordance with generally accepted methodological approaches. Research of cold and heat adaptation reactions of experimental animals have been conducted by using the model of a long cold and heat action with appropriate climatic chambers. In rats we were determined the physical performance (swimming, performance on the treadmill), biochemical values of the antioxidant activity of plasma to research the protective effect of a mixture of HP and RR during cold and heat influence [1].

The studied mixture (when it admission in doses of $150-300 \mathrm{mg} / \mathrm{kg}$ daily) increases the resistance animals to fatigue in the process of adapting to the cold and heat.

Thus, complex experimental researches give reason to recommend the powder blend of adaptogens from HP and RR as antihypoxic and anti-oxidants to prevent adverse effects of cold and heat stress on the body.

\title{
Literature
}

1.Korshunova, N. V. Dissertation of doctor of medicine: 14.00.07; St. Petersburg; 2001; 224.

2.Methodical instructions on the use design and rapid experimental methods under hygienic standardization of chemical compounds in water of water objects 1979; 26.

3.Methodical instructions on the study of the allergenic action for substantiation of maximum permissible concentrations of harmful substances in water of water bodies 1980; 17.

4.Pavlov, A.S. Fiziologiya cheloveka 2006; 32: 4: 110-115.

5.Nevmyvako E.E., Dorovskikh V.A., Korshunova, N.V., Shapovalenko N.S. Bulletin physiology and pathology of respiration 2011; (41):31-34.

6.Hyperforin production in Hypericum perforatum root cultures / M.Gaid [et al.] // J. Biotechnol. 2016. №222. P.47-55.

7.Hung S.K., Perry R., Ernst E. The effectiveness and efficacy of Rhodiola rosea L.: a systematic review of randomized clinical trials // Phytomedicine. 2011. Vol.18, №4. P.235-244.

DOI: 10.22448/AMJ.2016.15-16.82-84

UDC 618.3-06:616.248-08

\section{FEATURES OF CLINICAL COURSE, TREATMENT AND PREVENTION OF BRONCHIAL ASTHMA DURING PREG- NANCY AT THE MODERN STAGE}

Luchnikova T.A., Prikhodko O.B., Aistova M.V., Ryabukhina S.E.

Amur State Medical Academy, Blagoveshchensk, Russia, Amur Region Perinatal Centre, Blagoveshchensk, Russia, Women's consultation №1, Blagoveshchensk, Russia

Abstract The aim is to study the clinical course, the features of treatment of asthma in pregnant women and prevention of exacerbations. Having analyzed 85 pregnant women with asthma at various stages of gestation, it was found that the average duration of disease, was more than 10 years, with more frequent bronchial asthma mild persistent currents, allergic form. As for the level control, which at the moment makes the GINA (2016), pregnancy reduces it. Most pregnant women refer to a group with partially uncontrolled or uncontrolled asthma.

Key words: bronchial asthma, pregnancy, clinical course, treatment.

Bronchial asthma (BA) is one of the common diseases of the pulmonary system, and takes the first place in the structure of allergic diseases in pregnant women. According to the Institute of Immunology in Russian asthma suffer from $2 \%$ to $18 \%$ of the population. The prevalence of asthma among pregnant women in Europe is around $4 \%$, the morbidity of asthma among pregnant women in the Russian Federation range from $0.4-1.3 \%$ to $5.2 \%$. Consideration of mutual influence of bronchial asthma and pregnancy remains relevant in connection with growth of frequencies of asthma, high percentage of obstetric complications, adverse perinatal outcomes, high morbidity of newborns and children. Difficulty of asthma early detection in pregnancy and selection of therapy dictate the need for increased diagnostic capabilities and search tools for monitoring the effectiveness of treatment.

The aim of work - study of the clinical course, the features of treatment Asthma in pregnant women and prevention of exacerbations.

Material and methods. Have been analyzed the clinical course of asthma in 85 pregnant women with an average duration of disease, respectively, 9,7 $\pm 2,5$ years and 7,5 $\pm 2,3$ year. According to the perinatal center of Blagoveschensk, women's consultations of Blagoveschensk, city maternity home of Blagoveschensk.

Among the examined 85 pregnant women at $62.1 \%$ of the patients had Asthma prevailing light current 
(BALC), at 30.6\% - moderate (BAMC), at 8.3\% - heavy (BAHC), which reflects the general distribution structure Asthma severity among the population.

Family history for allergic diseases traced in $40.7 \%$ of patients, while at Asthma $-35.5 \%$ of them on the maternal side - at $59.2 \%$. In 15 patients with asthma observed in three generations, including through the mother - in 12 (80\%), on the father - in $3(20 \%)$.

The duration of Asthma up to 5 years were 33 patients (20.7\%), more than 5 years 52 (79.3\%), of whom more than 10 years - in 29 (45.9\%), more than 20 years - in 4 (16,9\%), over 30 years - in $1(3.4 \%)$. In $27(7.2 \%)$ patients had a history of asthma status, during which the two of them at gestation period 9 and 14 weeks has a spontaneous miscarriage.

In $42(62.4 \%)$ patients were diagnosed a form of allergic asthma, in 7 (10.4\%) - non-allergic, in $36(27.2 \%)$ mixed. It was revealed that the predominant BALC was allergic form ( $71.7 \%)$, with the BAMC and BAHC - non-allergic and mixed forms of the disease.

Aggravation of asthma during pregnancy was found in $65.2 \%$. In light current $-48.8 \%$ of patients, with moderate - from 70 to $81.4 \%$, with a heavy - at $100 \%$.

In patients with BALC for one trimester of gestation exacerbation was $59.3 \%$, preferably with an allergic form, respectively, I trimester $-17.4 \%$ in II $-31,6 \%$, in the III - 10,0 \%. Furthermore, $30.6 \%$ of patients repeatedly exacerbated asthma during pregnancy: at $57.9 \%$ of them - I and II trimester, in $18.4 \%$ - in the I and III trimester, at $31.0 \%$ - in II and III. Exacerbations of asthma were more frequent at the 12-13 and 18-20 weeks of pregnancy

Patients with BAMC exacerbation during one trimester was observed in $48.5 \%$ of pregnant women are more likely an allergic and mixed forms and rarely - at non-allergic. What is most likely characterized by receiving basic therapy during pregnancy, as opposed to women with BALC. Exacerbation of asthma for I and II trimesters was observed in $10.0 \%$ of women, I, and III trimesters of $-10.0 \%$, II and III trimester - at $22.9 \%$. In $17.1 \%$ of patients the disease exacerbation observed during all trimesters of pregnancy.

The most frequent asthma exacerbations were in the following terms of gestation 18-20 weeks, 25-28 weeks, 35-36 weeks. Exacerbation of asthma in this group of patients had 1.5 times more likely for two, three trimesters of pregnancy, than one, and it was more characteristic for non-allergic form of the disease.

In the group of patients with exacerbation BAHC of its during one trimester were $25 \%$ of patients at $30-32$ weeks gestation and 35-37 weeks. More than half of women exacerbation were followed for two trimesters: I and III $-16,7 \%$ of pregnant women, in II and III - 45,8\%, $12.5 \%$ cases were acute throughout pregnancy, then there, there was continuously relapsing course of asthma. In $3(12.5 \%)$ patients developed asthmatic status with gestation of 36 - 37 weeks.

In $11(13.1 \%)$ of patients first diagnosed asthma during pregnancy. At the same time, 8 of them are marked BAMC, allergic form, 3 - BALC, allergic form.

Patients were treated according to GINA guidelines (2014), depending on the disease control level and phase of the disease. During an exacerbation of asthma, most patients were hospitalized $(73.5 \%)$, the rest were treated as outpatients. In order to assess the level of asthma control using the "Test of asthma control" (ASTMM) in 80 patients. It was noted that only half of them have not experienced difficulties in carrying out normal workload, the symptoms observed 1 time per day during the last 4 weeks 40 (44.4\%) patients. At the same time, only $21(23.3 \%)$ of respondents did not use the $\beta 2$ - short-acting agonist for the study period (pic.10).

According to the latest recommendations of GINA [2] a pregnancy refers to the exacerbation factors, so the occurrence of pregnancy is necessary to continue the assigned therapy, including the following drugs: $\beta 2$ agonists, inhaled corticosteroids, montelukast, theophylline, and that these drugs are not associated with an increased risk of complications to the fetus in contrast to loss of control (level of evidence A). Even if patients have good control of the disease, it is recommended to assign them low-dose of inhaled corticosteroids (level of evidence A).

According to the latest recommendations of GINA [2] a pregnancy refers to modifying risk factors of asthma exacerbation. In order for pregnancy proceeded well, all women who suffer from of asthma, must take pregravid preparation for pregnancy, to be able to achieve of asthma control, selection of drugs capable of keeping a level of control throughout the pregnancy and explain patient with bronchial asthma about the need of therapy and increasing exacerbation risk when its abolition.

As for the best course of bronchial asthma during pregnancy except the basic treatment is necessary to carry out the primary prevention of bronchial asthma. It should reduce or avoid contact with inhaled allergens, to be vaccinated against influenza, because ARVI greatly increase the risk of exacerbation, taking vitamin $D$ and $\mathrm{E}$, which reduce the level of bronchial obstruction. Also contributes to the risk of exacerbation antibiotics. The social environment in which the child can also contribute to the development bronchial asthma and influence the degree of its severity. Stress of mother, which preserved from birth to primary school-age child has a relationship with an increased risk of asthma of children.

\section{Literature}


1.Dynamics of mild persistent bronchial asthma course in pregnant women / A.F. Babtseva, O.B. Prikhodko, E.B. Romantsova, Y.S. Landishev, I.V. Kostrova // Breath physiology and pathology Journal, 2012. Issue 46. P. 39-43.

2.Global Initiative for Asthma (GINA), 2014. 147 p.

3.Features of social-economic status in pregnant women with bronchial asthma / T.A. Luchnikova, O.B. Prikhodko // Breath physiology and pathology Journal, Blagoveshchensk, 2015. Issue 56. P. 78-82.

4. Evolution clinical and epidemiological course of bronchial asthma in during pregnancy / T.A. Luchnikova, O.B. Prikhodko, A.F. Babtseva, E.B. Romantsova, M.V. Pogrebnaya, E.I. Smorodina // The 11th Sino-Russia forum of biomedical and pharmaceutical science: the conference proceedings. Harbin, 2014. P.166-167.

5.Prikhodko O.B. Clinical and functional features of bronchial and lung systems and neuro-vegetative regulation in patient with asthma in during pregnancy and their effects on offspring. abstract of thesis...doctor of medical sciences. Blagoveshchensk, 2010. 44 p.

6.Prikhodko O.B. Neurovegetative regulation and hemodynamics of pregnant women with bronchial asthma // Allergology and Immunology Journal. 2009. V. 10, № 1. P. 84.

7.The role of controlled asthma in the development of complications of pregnancy and influence on the health of newborns / O.B. Prikhodko, A.F. Babtseva, E.B. Romantsova //International journal on immunorehabilitation. 2009. V. 11, № 1. P. 38-39.

8.Influence of clinical and functional features of bronchopulmonary system at pregnant women with asthma on the condition of child /O.B. Prikhodko, A.F. Babtseva, E.B. Romantsova//International journal on immunorehabilitation. 2010. V. 12, № 2. P.114a.

9. The lack of asthma control during pregnancy as a predictor of perinatal complications / O.B. Prikhodko, A.F. Babtseva, E.B. Romantsova, Y.S. Landyshev, T.A. Luchnikova, E.I. Smorodina, I.V. Kostrova //Allergology and Immunology Journal. 2013. V. 14, № 3. P.188-189.

10.Trofimov V.I. Features of the course and treatment of asthma in pregnant women // New St. Petersburg medical statements. 2009. №2. P.34-37.

11.The clinical course of asthma in pregnant smokers / A.S. Zenkina, O.B. Prikhodko, A.F. Babtseva, E.B. Romantsova // Materials of the VI congress of pulmonologists of Siberia and Far East. Blagoveshchensk, 2015. P.36-39.

\section{DOI: 10.22448/AMJ.2016.15-16.84-85 \\ UDC 618-01 \\ ANGIOGENIC FACTORS IN WOMEN WITH EXCESSIVE MENSTRUATION IN PUBERTY \\ Lysyak D.S.}

\section{Amur State Medical Academy, Blagoveshchensk, Russia}

Abstract. $10 \%$ of women with excessive menstruation in puberty in reproductive age are saved from heavy and prolonged menstruation. It is accompanied by increased expression of VEGF by 1.5 times and the development of excessive angiogenesis in the endometrium.

Key words: puberty bleeding, vascular endothelial growth factor.

Excessive menstruation in puberty (EMP) account for $50 \%$ of complaints girls-teenagers to the gynecologist. This pathology of puberty may be the first clinical manifestation of the emerging gynecological pathology $[1,2]$. With timely treatment of EMP correct menstrual rhythm in reproductive age is set in $82.9 \%$ of patients, in $10 \%$ - heavy menstruation [3]. One of the components of the pathogenesis of endometrial hyperplasia and uterine bleeding in teenagers is a high level of IGF-1 in combination with decreased production of VEGF and activation of the hypothalamic-pituitary-adrenal system. In patients with oligomenorrhea and hyperandrogenic ovarian dysfunction by increasing the content of IGF-1 retained adequate production of VEGF, indicating that the stored angiogenic potential [4]. In women with EMP the rate of spontaneous abortion in early gestational period is reaches $4 \%$ [5].

The purpose of the study was to evaluate the angiogenic effects in women of reproductive age with EMP in the period of an implantation window.

Material and methods. The study included 60 patients, who were divided into two groups: the study group consisted of 30 patients with EMP, the control group - 30 patients with the correct timing of menstruation from menarche period. All patients had a two-phase menstrual cycle is established by ultrasound. Exclusion criteria were: overweight, obesity, early age at menarche (under 11 years), hyperandrogenism, confirmed by clinical and hormonal studies, diseases of the reproductive system organs, using oral contraceptives the last 3 months before the examination.

The concentration of vascular endothelial growth factor (VEGF) and its receptor (VEGF-R1, VEGF-R2) were determined in venous blood serum on 20-24 days of the menstrual cycle. 